\title{
Bann en créole mauricien : nom de quantité et morphème de pluralité
}

\author{
Alleesaib, Muhsina \\ Université Paris 8, Saint-Denis \\ UMR 7023, ED CLI \\ muhsina.alleesaib@sfl.cnrs.fr
}

\section{Données et analyses précédentes}

Cette étude est consacrée à un morphème analysé comme morphème de pluralité et qui détermine le nom en créole mauricien. On retrouve le marqueur de nombre bann dans trois créoles français : le mauricien, le réunionnais et le seychellois (Baker 1972,2003; Corne 1970; Bollée 1977, Staudacher-Valliamée 2004). Les exemples suivants en illustrent l'emploi en mauricien :

$\begin{array}{lllll}\text { a. } & \text { Butej } & \text { la } & \text { ranpli. } & \\ & \text { bouteille } & \text { DEF } & \text { plein } & \\ \\ \text { 'La bouteille est pleine.' } & & \\ \text { b. } & \text { Bann } & \text { butej } & \text { la } & \text { ranpli. } \\ & \text { BANN } & \text { bouteille } & \text { DEF } & \text { plein }\end{array}$

'Les bouteilles sont pleines.'

Il existe deux prononciations concurrentes de bann en mauricien contemporain : /ban/ ou /ben/. Dans les sous-sections suivantes présentent les précédentes analyses de bann dans les créoles français des Mascareignes.

Le créole mauricien est un créole à base lexicale française parlé à l'Ile Maurice et qui est très proche des créoles parlés sur l'Ile Rodrigues et dans les Seychelles. La population de l'Ile Maurice est d'environ 1.2 million. Le créole mauricien est la langue vernaculaire pour une majorité de Mauriciens qui maîtrisent aussi d'autres langues à des degrés divers. Les langues les plus employées à l'écrit sont l'anglais et le français, les langues coloniales qui sont aussi les langues de la scolarisation. Le français et le créole sont les deux langues dominantes dans les médias. Le créole mauricien est en voie de standardisation, mais reste une langue majoritairement orale.

\subsection{Précédentes analyses de bann}

Les analyses qui en ont été faites portent sur trois points: l'optionalité apparente, le processus de 'grammaticalisation', les effets de définitude.

Le fait que bann n'apparaît pas systématiquement dans des contextes où, dans d'autres langues, la marque du pluriel serait obligatoire a motivé une analyse en termes d'optionalité, selon laquelle bann exprime la pluralité et est employé soit si le contexte le demande, soit afin de souligner le caractère pluriel du référent (Baker 1972). Lorsqu'il décrit les occurrences de bann dans les trois créoles en question, Baker (2003) ne donne pas plus de détails sur les conditions de cette optionalité. 
(2) MAU (bann) lakaz la 'la/les maison(s)'

$$
\begin{array}{ll}
\text { SEY } & \text { sa (bann) lakaz } \\
\text { REU } & \text { le (bann) lakaz }
\end{array}
$$

(adapté de Baker $2003: 139)$

En seychellois, Bollée (1977) suggère que l'emploi de bann est restreint aux référents spécifiques tandis qu'en réunionnais, Chaudenson (2003) note que bann est peu employé à l'écrit, mais beaucoup à l'oral. Je m'attacherai à décrire le fonctionnement particulier de bann en mauricien, et je ne dirai rien sur son fonctionnement dans les autres créoles à base française de l'Océan Indien.

Bann est généralement classé parmi les déterminants prénominaux, étant donné qu'il commute avec enn 'un'.

$$
\begin{array}{llllll}
\text { Donn } & \text { nu } & \text { enn/ } & \text { bann } & \text { bon } & \text { nouvel. } \\
\text { donner } & 1 \mathrm{PL} & \text { un } & \text { BANN } & \text { bon } & \text { nouvelle }
\end{array}
$$

'Donne(z)-nous \{ une / de \} bonne(s) nouvelle(s).'

Il peut être commuté avec les cardinaux (un, deux, trois), avec l'adverbe de quantité buku, ou avec les morphèmes de quantité imprécise (plizir, sak). Cependant, il apparaît toujours à droite du démonstratif : sa bann butej la 'ces bouteilles'.

La question centrale de ce présent travail est la distribution et les effets produits par l'apparition de bann. Le cadre théorique sera celui des Principes et Paramètres de la grammaire générative. Dans un premier temps, je propose de vérifier la compatibilité des deux types de nominaux avec des prédicats appelant un sujet au pluriel.

\subsection{Des référents pluriels}

Les référents pluriels sont compatibles avec des prédicats du type se réunir, se rassembler, se regrouper qui exigent d'avoir un sujet pluriel.

$\begin{array}{lllll}\text { a. Bann zanfan inn fer letour } & \text { zot } & \text { mama } \\ \text { BANN enfant ACC faire tour } & \text { POSS-3PL } & \text { mère } \\ \text { 'Les enfants ont entouré leur mère.' } & & \\ \text { b. } \quad \text { Contexte: D'habitude, à l'heure du repas] } & & \\ & \text { Zanfan fer letur zot/ } & \text { *so } & \text { mama } \\ \text { enfant faire tour POSS-3PL } & \text { POSS-3SG } & \text { mère } \\ \text { (i) 'Les enfants entourent leur mère.' } & & \\ \text { (ii) *'L'enfant entoure sa mère.' }\end{array}$

Suivant ce diagnostic, bann $+\mathrm{N}$ désigne effectivement un référent pluriel. Le fait remarquable est qu'en (4b), le nom nu désigne aussi selon le test un référent pluriel. Je propose d'aborder la question de la distribution des constructions bann $+\mathrm{N}$ en les confrontant avec les noms nus dans les mêmes contextes. A partir de là, je prédirai que les noms nus ne produisent pas les mêmes effets de sens que bann $+\mathrm{N}$. Il sera question de l'interprétation pluriel des $\mathrm{SN}$ contenant bann $^{\mathrm{PL}}$ dans la section 3 . 
Ce travail sera divisé en deux parties : la première concernera la division de bann en trois items différents, selon des critères syntaxiques et sémantiques.

- un nom lexical bann ${ }^{\mathrm{NL}}$, dont le sens est 'groupe',

- une expression de quantité $b a n n^{\mathrm{NQ}}$ qui apparaît dans l'expression de quantité enn bann,

- une marque de pluriel bann ${ }^{\mathrm{PL}}$.

Dans la seconde partie, je m'attarderai sur quelques effets interprétatifs produits par bann ${ }^{\mathrm{PL}}+\mathrm{Nom}$.

\section{Polyfonctionnalité de bann en mauricien contemporain}

Les sous-sections suivantes présentent des critères syntaxiques qui permettent d'isoler les trois types d'emploi de bann.

\section{$2.1 \quad$ bann $^{N L}$ comme nom lexical}

Dans cette section, il est question tout d'abord de la distribution de bann en tant que nom lexical ; la suite de la section traite des effets interprétatifs dont je postule qu'ils sont liés à cet emploi particulier de bann.

\subsection{1 bann ${ }^{N L}$ 'groupe' : désigne une classe ou un ensemble d'entités.}

Cet emploi de bann est celui d'un nom lexical : il appartient à une classe ouverte et peut commuter avec le nom group 'groupe'. Il s'emploie avec un nom commun pour désigner un groupe. En tant que nom lexical, bann $^{\mathrm{NL}}$ est compatible avec un cardinal (5b) et peut être modifié par un adjectif (5c).

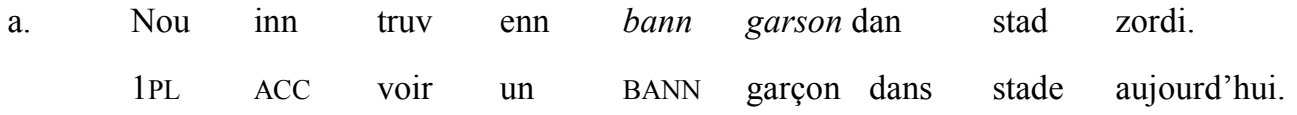

'Nous avons vu un groupe de garçons au stade aujourd'hui.'

b. Nou inn truv trwa bann garson dan stad zordi.

1PL ACC voir trois BANN garçon dans stade aujourd'hui.

'Nous avons vu trois groupes de garçons au stade aujourd'hui.'

$\begin{array}{lllllll}\text { c. Enn } & \text { gran } & \text { bann } & \text { zanfan } & \text { ti } & \text { pe } & \text { zwe. } \\ \text { Un } & \text { grand } & \text { BANN } & \text { enfant } & \text { PASS } & \text { PROG } & \text { joue }\end{array}$

'Une grande bande d'enfants était en train de jouer.'

Des synonymes de cet emploi particulier de bann sont larme 'gang' ou korom 'cohorte'. La structure cidessous représente l'analyse de bann ${ }^{\mathrm{NL}}$ qui prend un complément garson qui est aussi un NP. 


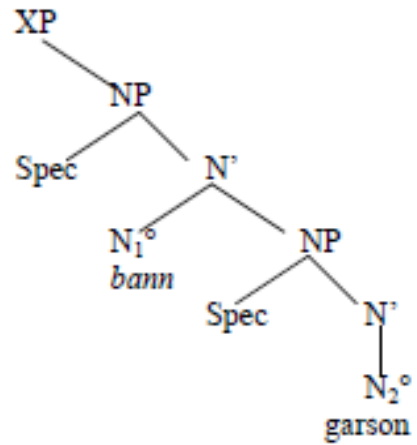

Une analyse plus approfondie de ce nom et de sa substituabilité avec les autres noms similaires est nécessaire, mais elle nécessite pour cela des données plus complètes. La section suivante traite de l'emploi de bann et la dénotation 'catégorie'.

\subsubsection{Bann ${ }^{\mathrm{NL}}+$ Nom désigne une classe d'objets}

L'emploi suivant de bann est classé parmi les emplois du type 'groupe', dans la mesure où je postule que l'idée de catégorie d'objets n'en est pas très éloignée.

$$
\begin{array}{lllllll}
\text { a. Bann } & \text { zoranz } & \text { sidafrik } & \text { pli } & \text { bon } & \text { ki } & \text { tu. } \\
\text { BANN } & \text { orange } & \text { Afrique du Sud } & \text { plus } & \text { bon } & \text { que } & \text { tout }
\end{array}
$$

'Les oranges qui viennent d'Afrique du Sud sont meilleures (que toutes les autres).'

$\begin{array}{llllll}\text { b. } \quad \text { Zoranz sidafrik } & \text { pli } & \text { bon } & \text { ki } & \text { tu. } \\ \text { orange Afrique du Sud } & \text { plus } & \text { bon } & \text { que } & \text { tout }\end{array}$

'Toute orange venant d'Afrique du Sud est meilleure que les autres.'

Bann $^{\mathrm{NL}}+$ Nom et le nom nu peuvent tous deux apparaître dans ce contexte: je propose de les distinguer en termes de généralité. Le nom nu reçoit une interprétation de nom d'espèce ou nom générique (7b), tandis que le SN avec bann en (7a) dénote l'ensemble des unités qui constituent la dénotation de zoranz sidafrik, c'est-à-dire l'extension de cette expression (Arrivé et al. 1986). Afin de justifier cette distinction, il est utile de recourir à un contexte. Si l'on imagine qu'à la suite d'une catastrophe écologique, l'Afrique du Sud ne produise plus d'oranges, la proposition en (7a), avec bann, serait bizarre, mais on pourrait toujours dire (7b), qui ne présuppose pas l'existence, au moment de l'énonciation, des oranges sud-africaines. De plus, dans le cadre d'un concours d'oranges, si quelqu'un goûte deux exemplaires d'oranges sud-africaines parmi plusieurs sous-espèces d'oranges, il pourrait prononcer (7a) mais pas (7b), la raison étant que deux exemplaires ne constitue pas un nombre suffisant pour généraliser sur toute une espèce.

\subsubsection{L'emploi 'pluriel associatif' de bann ${ }^{\mathrm{NL}}$}

Le terme 'pluriel associatif' est employé lorsqu'on désigne un groupe d'individus associés à une personne ou originaires d'un lieu.

Bann $^{\mathrm{NL}}$ est employé avec un nom de personne (8) et avec un nom de lieu (9). 
(8)
Mo inn trouv bann
voir BANN
Zidane jer.
$1 \mathrm{SG}$
$\mathrm{ACC}$
Zidane hier
(i) 'J'ai vu la bande à Zidane hier.'
(ii) *'J'ai vu une bande à Zidane hier.'

Le fait de l'employer avec un nom de personne produit l'interprétation 'amis', 'collègues' ou 'famille' de cette personne.

$\begin{array}{llllll}\text { Mo } & \text { inn } & \text { trouv } & \text { bann } & \text { Moka } & \text { jer. } \\ \text { 1SG } & \text { ACC } & \text { voir } & \text { BANN } & \text { Moka } & \text { hier }\end{array}$

(i) 'J'ai vu la bande de Moka hier.' (ex. nos amis de Moka)

(ii) *'J'ai vu une bande de Moka hier.'

Avec le nom de lieu Moka, bann ${ }^{\mathrm{NL}}$ renvoie à un groupe d'individus [+humain] originaires ou résidents de ce lieu. Les NP à interprétation 'pluriel associatif' sont toujours définis, cet effet étant probablement dû à la présence du nom propre qu'ils contiennent.

En résumé, la section 2.1 traite d'un ensemble d'emplois de bann que j'ai regroupé sous l'étiquette de l'emploi de bann comme nom lexical. Les SN contenant bann ${ }^{\mathrm{NL}}$ sont analysés comme des nominaux complexes.

\subsection{Bann dans l'expression de quantité enn bann : bann ${ }^{\mathrm{NQ}}$}

\subsubsection{Structure des DP avec enn bann}

L'arrière-plan théorique adopté ici est la structure en DP. Selon l'hypothèse DP (Abney 1987, inter alii), les syntagmes nominaux doivent être représentées par une projection DP (Determiner Phrase) où se trouve le déterminant. Les déterminants démonstratifs et la marque de défini la occupent cette projection.

Borer (2005) enrichit la structure du DP en postulant des projections intermédiaires entre le DP et le NP : un syntagme de quantité (Quantity Phrase) et un syntagme de nombre (Number Phrase).Le NumP comprend la morphologie du pluriel ou les classificateurs (dans les langues à classificateurs généralisés) ; sa fonction sémantique lorsqu'il est projeté est d'opérer une division du référent en unités dénombrables.

Le QtP est projeté plus haut que le NumP et attribue une quantité (six, plusieurs) aux unités créées par ce dernier. Ainsi three cats 'trois chats' donne la représentation suivante : [QtP three [NumP $-\mathrm{S}$ [NP cat]]], où la morphologie du pluriel est sous NumP et le cardinal sous QtP.

Mon hypothèse est que enn bann se trouve dans le syntagme de quantité, dans la position de spécificateur et non en position de tête. 


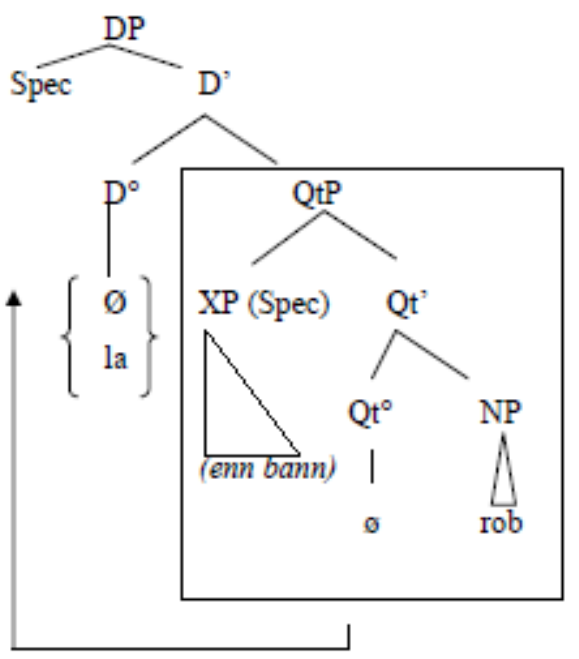

Enn bann est un syntagme placé dans le Spécificateur de QtP et produit l'interprétation de quantité. En raison de la position du défini LA à la droite du DP, je postule que le syntagme de quantité se déplace au niveau phonologique (en PF, Phonolgical Form) dans le Spec de DP. Cette analyse est reprise de ZribiHertz et Glaude (2007).

Bann ${ }^{\mathrm{NQ}}$ forme avec enn 'un' une expression de quantité enn bann lit. 'une bande de', signifiant 'une quantité de'. Les expressions de quantité sont formés d'un déterminant et d'un nom. Ce type d'expression se retrouve par exemple en français et en anglais.

(11) un tas de, un amas de, une quantité de,

(12) a bunch of 'un groupe de, une grappe de' et a lot of 'beaucoup de' .

En plus de enn bann, nous trouvons les expressions suivantes : enn pake ' un paquet de', enn ta 'un tas de', enn vole 'une volée de', enn kantite 'une quantité de'.

$\begin{array}{llllllll}\text { (13) Nou- } & \text { inn } & \text { truv } & \text { enn } & \text { bann } & \text { garson dan } & \text { stad } & \text { zordi. } \\ \text { 1PL } & \text { ACC } & \text { voir } & \text { un } & \text { BANN } & \text { garçon dans } & \text { stade } & \text { aujourd'hui }\end{array}$

'Nous avons vu un tas de jeunes hommes au stade aujourd'hui.'

Les exemples en (14) illustrent le fait que, contrairement à certains emplois de bann $^{\mathrm{NL}}$, bann ${ }^{\mathrm{NQ}}$ ne peut être modifié (14a)(ii), et qu'il forme une expression figée avec enn. Ce type de bann n'est donc pas à traiter comme un nom commun. Il en est de même avec les autres expressions de quantité enn pake et enn $\operatorname{ta}(14 \mathrm{~b})$.

$\begin{array}{lllllll}\text { a. Enn } & \text { gran } & \text { bann } & \text { zanfan ti } & \text { pe } & \text { zwe. } \\ \text { un } & \text { grand } & \text { BANN } & \text { enfant } & \text { PASS } & \text { PROG } & \text { joue }\end{array}$

(i) 'Une grande bande d'enfants était en train de jouer.' ( ${ }^{\mathrm{OK}}$ bann ${ }^{\mathrm{NL}}$ )

*'Une grande quantité d'enfants était en train de jouer.'( $\left.{ }^{*} b a n n{ }^{\mathrm{NQ}}\right)$ 


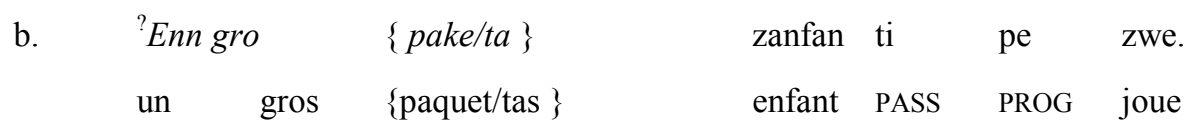

Lit. 'Un gros paquet/tas d'enfants était en train de jouer.' ( ${ }^{\mathrm{OK}}$ mais étrange)

*'Une grande quantité d'enfants était en train de jouer.'

\subsubsection{Différences entre enn bann et les autres expressions de quantité}

Enn bann se distingue des autres expressions de quantité par le fait qu'il produit une lecture discontinue du référent (15b), ce qui n'est pas le cas pour les autres expressions (15a).

a. Ena $\quad\{$ enn ta/ enn pake $\}$ lapusjer anba larmwar.
avoir $\{$ un tas / un paquet\}poussière

'Il y a un tas (beaucoup) de poussière sous l'armoire.'

$\begin{array}{lllll}\text { b. Ena enn } & \text { bann } & \text { lapusjer anba larmwar. } \\ \text { avoir un } & \text { BANN } & \text { poussière } & \text { sous armoire }\end{array}$

Lit. 'Il y a beaucoup de poussières (dispersées) sous l'armoire.'

Avec des référents strictement continus comme le sable ou la boue, l'emploi de enn bann est exclu en (16a), mais d'autres expressions de quantité peuvent être employées, à condition de permettre une lecture massique du référent.

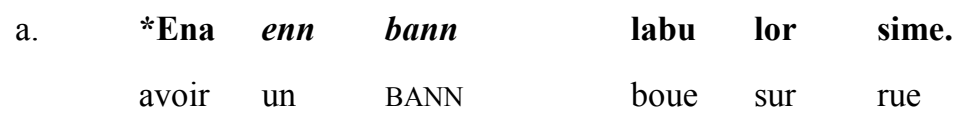

Lit 'Il y a un tas de boues dans la rue.'

$\begin{array}{lllllll}\text { b. Ena }\{\text { enn } & \text { ta } / & \text { enn } & \text { pake }\} & \text { labu } & \text { lor } & \text { sime. } \\ \text { avoir }\{\text { un tas } / \text { un } & \text { paquet }\} \text { boue } & \text { sur } & \text { rue. }\end{array}$

'Il y a un tas de boue dans la rue.'

Ces faits suggèrent l'hypothèse suivante : l'effet sémantique discontinu induit par enn bann a favorisé le développement de bann comme marque de pluriel.

Un autre point de différence entre enn bann et les autres expressions de quantité disponibles en CM concerne les compléments circonstanciels. Selon Doetjes (1997), un Quantifieur + Nom en position de circonstant permet la quantification indirecte de l'événement décrit par le verbe. Cette quantification indirecte du domaine verbal n'est pas disponible pour enn bann (voir l'exemple 17b).

Certaines expressions de quantité, par exemple enn pake (lit. 'un paquet'), enn kantite ('une quantité'), enn ta ('un tas'), peuvent quantifier un nom, formant ainsi un complément circonstanciel de temps, mais pas enn bann. En (17a), le complément circonstanciel peut être enn pake 'beaucoup' ou enn pake fwa 'plusieurs fois'. En (17b), ni enn bann ni enn bann fwa ne peuvent former un complément circonstanciel. 


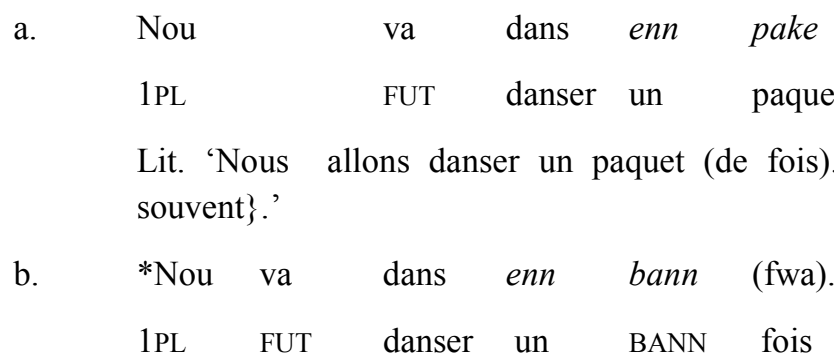

Lit. 'Nous allons danser une quantité (de fois).'

A partir de ce contraste, on peut supposer que enn bann peut quantifier sur des entités, mais pas sur des événements. Cette contrainte a pu favoriser le développement de bann comme marque de pluriel; elle pourrait aussi expliquer pourquoi les autres expressions de quantité n'ont pas permis ce développement.

Dans la représentation en (18), le déterminant défini LA est représenté dans le même syntagme que enn bann, suggérant qu'ils sont compatibles. De ce fait, l'exemple suivant illustre la compatibilité de l'expression de quantité avec le défini la: celui-ci est employé si le référent 'une quantité de robes' a déjà été mentionné. Cette combinaison n'est pas permise en français.

$\begin{array}{lll}\text { Mo } & \text { inn } & \text { aste } \\ 1 \mathrm{SG} & \text { ACC } & \text { acheter }\end{array}$
enn bann rob
(la) jer.
'J'ai acheté une quantité de robes hier.'
'J'ai acheté la quantité de robes (préidentifiée) hier.'
b. J'ai acheté (*la) une quantité de robes hier.

Un dernier aspect de la syntaxe des nominaux avec Bann $^{N Q}$ concerne leur compatibilité avec les lectures téliques.

$\begin{array}{llllllll}\mathrm{Li} & \text { mont } & \text { enn } & \text { bann } & \text { lakaz } & \text { dan } & \text { enn } & \text { lane. } \\ \text { 3SG } & \text { construire } & \text { un } & \text { BANN } & \text { maison } & \text { dans } & \text { un année } \\ \text { 'Il a construit un tas de maisons en un an.' } & & \end{array}$

Après cette présentation des emplois de bann en tant que nom (plein ou à l'intérieur d'une expression de quantité), la prochaine section traite du bann en tant que déterminant exprimant la pluralité.

\subsection{La marque de pluralité : Bann ${ }^{\mathrm{PL}}$}

Contrairement aux précédentes analyses de ce morphème, j'émets ici l'hypothèse que bann ${ }^{\mathrm{PL}} \mathrm{n}^{\text {'est }}$ pas optionnel. Cette affirmation se base sur le postulat que, dans une grammaire donnée, si un élément produit des effets sémantiques qui sont absents lorsqu'il n'apparaît pas, cet élément ne peut être décrit comme optionnel.

Dans la présente section, je mets en avant les divers effets sémantiques déclenchés par bann ${ }^{\mathrm{PL}}$, afin d'argumenter contre l'hypothèse de l'optionalité de bann. 


\subsubsection{La différence entre le nom nu et bann ${ }^{\mathrm{PL}}+\mathrm{N}$}

Selon de nombreux auteurs, dont Borer (2005) et Kwon \& Zribi-Hertz (2004), le nom nu est neutre et non-atomisé du point de vue la grammaire (indépendamment de la nature du référent). Lorsqu'il apparaît, bann ${ }^{\mathrm{PL}}$ signale que le référent désigné par le nom est au nombre de 'au moins 2'. La différence entre $b_{a n n}{ }^{\mathrm{PL}}+\mathrm{N}$ et le nom nu concerne l'absence de spécification de nombre et une spécification de pluriel. Je souligne ici que le nom non marqué en CM n'a pas la valeur de singulier (cf. (4b) plus haut).

$$
\begin{array}{llll}
\text { a. } & \text { Inn } & \text { gagn } & \text { aksidan gramatin. } \\
& \text { ACC } & \text { arriver } & \text { accident matin }
\end{array}
$$

'Il s'est produit (un ou plusieurs) accident(s) ce matin.'

$\begin{array}{llll}\text { b. Inn gagn bann aksidan } & \text { gramatin. } \\ & \text { ACC } & \text { arriver BANN accident } & \text { matin }\end{array}$

'Il s'est produit des accidents ce matin.'

En (20a), le nombre d'accidents n'est pas spécifié, et en (20b), il y en a plusieurs.

\subsubsection{Bann ${ }^{\mathrm{PL}}$ bloque la lecture massique}

Cette analyse présuppose que les noms ne sont pas préalablement marqués en tant que massiques ou comptables dans le lexique. Les interprétations qu'ils reçoivent dépendent de la structure syntaxique (emploi d'un classificateur ou morphologie du pluriel) dans laquelle ils sont enchâssés. Lorsqu'un nom est employé sans aucune marque, il peut recevoir une lecture massique, dépendant de la nature du référent qu'il désigne. Cette lecture massique possible disparaît avec l'emploi de bann.

Bann ${ }^{\mathrm{PL}}$ partage avec les classificateurs dans les langues à classificateur généralisé la propriété de produire un effet d'atomisation du référent. L'emploi de bann ${ }^{\mathrm{PL}}$ induit une lecture en unités discrètes : on obtient ainsi une interprétation 'poussières dispersées' en (21a), 'différents morceaux ou différents types de viande en (22a) et 'différentes instances de pluie' en (23a).

$$
\begin{array}{llllllll}
\text { a. } & \text { Mo } & \mathrm{nn} & \text { balje } & \text { bann } & \text { lapusjer } & \text { anba } & \text { larmwar. } \\
& 1 \mathrm{SG} & \text { ACC } & \text { balayer } & \text { BANN } & \text { poussière } & \text { sous } & \text { armoire }
\end{array}
$$

\section{bann lapusjer}

b.

$\begin{array}{lllll}\text { Mo } & \text { inn } & \text { balje } & \text { lapusjer anba } & \text { larmwar. } \\ \text { 1SG } & \text { ACC } & \text { balayer } & \text { poussière } & \text { sous armoire }\end{array}$




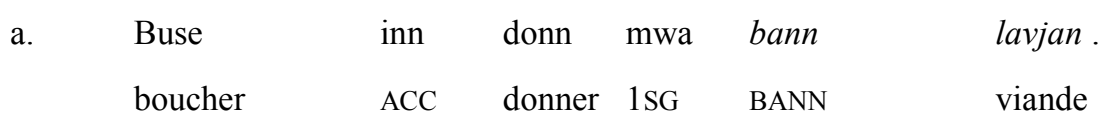

Lit. 'Le boucher m'a donné des viandes. = Le boucher m'a donné \{différents types/ des morceaux de viande/*de la viande.\}'

b. Buse inn donn mwa lavjan .

boucher ACC donner $\quad 1$ SG viande

'Le boucher m'a donné de la viande.'

$\begin{array}{cccccc}\text { a. } & \text { Nou } & \text { gagn } & \text { bann } & \text { lapli } & \text { lokalize. } \\ \text { 1PL } & \text { avoir } & \text { BANN } & \text { pluie } & \text { localisé }\end{array}$

'Nous avons \{des pluies localisées / *de la pluie localisée\}.'

b. Nou gegne lapli lokalize.

1PL avoir pluie localisé

'Nous avons de la pluie localisée.'

Je supposerai dans mon analyse que cet effet d'atomisation de bann ${ }^{\mathrm{PL}}$ est inhérent au morphème luimême, et pas le fait d'une tête fonctionnelle comme le suggère Borer (2005). La projection NumP, dont le rôle consiste à produire l'atomisation du référent en syntaxe, n'est pas nécessaire à l'architecture du DP en $\mathrm{CM}$.

\subsubsection{Certains contextes requièrent que le nombre soit spécifié}

Dans les langues où marquer le nombre n'est pas systématique, certains types de référents reçoivent une marque de nombre plus que d'autres (Corbett 2000 : 279-280). Par exemple, dans une langue donnée, les référents animés et les nominaux définis seront marqués, mais pas les référents inanimés et les indéfinis.

En $\mathrm{CM}$, les référents [+humain] et [+défini] pluriels sont marqués par bann $^{\mathrm{PL}}$ : en l'absence de marque, ces nominaux sont interprétés comme singulier, rendant ainsi bann ${ }^{\mathrm{PL}}$ nécéssaire.
a. $\quad$ Profeser
la anretar.
professeur
DEF
en retard

'Le professeur est en retard.'

[singulier/*neutre]

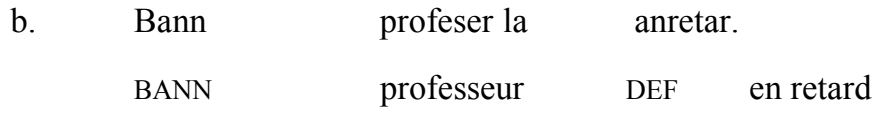

'Les professeurs sont en retard.' [pluriel] 


\subsubsection{Structure des DP avec bann ${ }^{\mathrm{PL}}$}

Voici une représentation arborescente des DP pluralisés.

$$
\text { bann rob (la) '(les) robes' }
$$

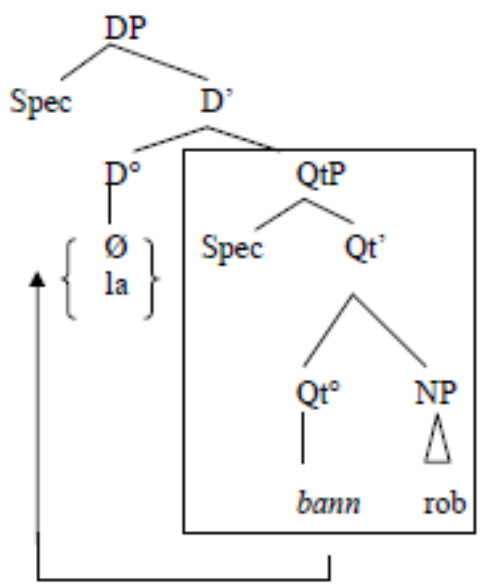

Je suppose ici que bann ${ }^{\mathrm{PL}}$ est la tête de la QtP, de même que l'expression de pluralité enn bann, tout en étant autonome. Ainsi que je l'ai suggéré plus haut, une projection de nombre (NumP si l'on suit les propositions de Borer 2005) ne s'applique pas en CM. La fonction de division du référent est remplie par bann $^{\mathrm{PL}}$.

A mon avis, l'argument suivant peut jutifier l'analyse de bann ${ }^{\mathrm{PL}}$ comme une tête fonctionnelle. Le fait que bann ${ }^{\mathrm{PL}}$ ne peut être modifié par un adjectif permet de le considérer comme une tête de syntagme.

$$
\begin{array}{ccll}
* \text { Mo inn avoy gran bann mesaz lor internet. } \\
\text { 1SG ACC envoyer grand BANN } & \text { message sur internet }
\end{array}
$$

(i) *'J'ai envoyé grand des messages sur internet. $\left(\right.$ bann $\left.^{P L / N Q}\right)$

(ii) ??'J'ai envoyé un grand groupe de messages sur internet.'( bann $^{\mathrm{NL}}$ )

L'exemple (26) illustre le fait qu'il n'est pas possible de modifier le bann de pluralité, mais que cela est possible avec l'emploi de bann comme nom. L'exemple en (27) et ses interprétations disponibles illustrent un aspect de la sémantique des bann ${ }^{{ }^{\mathrm{PL}}+}$ Nom : ces syntagmes peuvent soit recevoir une interprétation indéfini faible (à portée étroite), soit une interprétation définie (à portée large).

$$
\begin{array}{llllll}
\text { Bann } & \text { zelev } & \text { inn } & \text { reini } & \text { dan } & \text { lakour lekol. } \\
\text { BANN élèves } & \text { ACC } & \text { rassembler } & \text { dans } & \text { cour école }
\end{array}
$$

(i) (Contexte: Où sont les élèves ? )

'Les élèves se sont rassemblés dans la cour de l'école.' [lecture topique]

(ii) (Contexte : - 'Quelque chose a dû se passer.)

'Des élèves se sont rassemblés dans la cour de l'école.' [indéfini faible]

Bann ${ }^{\mathrm{PL}}$ diffère sur ce point des marqueurs de pluriel se (ANT) et yo (HAI), dont l'interprétation est strictement définie. Ceci est un constat intéressant pour la comparaison des créoles français. On peut 
illustrer plus avant les deux interprétations de $b a n n^{\mathrm{PL}}+\mathrm{Nom}$ en les insérant dans des phrases contenant d'autres syntagmes nominaux. Dans l'exemple (28), le SN bann zelev est mis en rapport avec des noms propres.

$$
\begin{array}{lllllll}
\text { Sam, } & \text { Anil } & \text { ek } & \text { Raj } & \text { inn } & \text { kessionnbann } & \text { zelev. } \\
\text { S. } & \text { A. } & \text { et } & \text { R. } & \text { PERF } & \text { interroger } & \text { BANN élève }
\end{array}
$$

(i) 'Sam, Anil et Raj ont interrogés les étudiants.'

(ii) 'Sam, Anil et Raj ont interrogés des étudiants (différents).'

(iii) *`Certains étudiants ont été interrogés par Sam, Anil et Raj.'

Une première interprétation de l'exemple (28), on présuppose l'existence d'un ensemble d'étudiants, dont on dit qu'ils ont été interrogés par trois individus. Le SN défini bann zelev est interprété comme un groupe. Lorsque l'interprétation de bann zelev est indéfini faible, l'interprétation gloable est distributive parce que le SN est sous la portée du SN sujet. L'interprétation en (28iii), où le SN bann zelev est un indéfini qui a portée large par rapport au SN sujet, est illégitime. Nous pouvons en conclure que si $b a n n^{\mathrm{PL}}+$ Nom est indéfini, il a toujours une portée étroite.

Ces quelques remarques soulignent la nécessité d'une étude plus approfondie des effets de définitude liés à la présence de bann.

\section{Effets interprétatifs des SN contenant bann ${ }^{\mathrm{PL}}$}

Dans cette partie, nous regardons de plus près les possibilités d'interprétation collective et/ou distributive des SN contenant bann. Je présente des arguments qui remettent en question l'assertion de Déprez (2009) selon laquelle ces SN sont interprétés de préférence de manière collective. Les données présentées plus bas indiquent qu'ils ressemblent comme les noms pluriels dans d'autres langues en ce que leur interprétation varie entre 'somme' et 'groupe'.

La thèse de Déprez (2009) s'appuie sur l'interprétation collective de la phrase suivante, qui, selon ses informateurs, est l'interprétation qu'ils préfèrent.

$\begin{array}{llllll}\text { Bann } & \text { zanfan } & \text { ti } & \text { sant } & \text { enn } & \text { sante. } \\ \text { BANN } & \text { enfant } & \text { PASS } & \text { chanter } & \text { un } & \text { chanson }\end{array}$

(i) 'Les enfants ont chanté une chanson (en chœur).'

(ii) 'Les enfants ont chacun chanté une chanson.'

L'interprétation collective signifie que les enfants ont chanté en chœur et l'interprétation distributive, que chaque enfant a chanté une chanson séparément. Selon l'auteur, l'interprétation (ii) est exclue, une intuition que je ne partage pas. L'interprétation distributive est aussi disponible.

Un des moyens de vérifier la disponibilité de l'interprétation distributive est de combiner un SN avec des prédicats dont le sujet doit être un individu. C'est le cas des prédicats dénotant des professions, comme l'illustre la phrase en (30).

(30) Les sœurs Brontë étaient des écrivains. 
Le prédicat être écrivain ne s'applique qu'à des individus distincts : la phrase en (30) signifie que chacune des sœurs Brontë était écrivain. Par ailleurs, il existe des contrastes entre les SN qui dénotent des groupes et les $\mathrm{SN}$ coordonnés. L'objectif est de comparer $b a n n^{\mathrm{PL}}+\mathrm{Nom}$ aux deux types de $\mathrm{SN}$ afin de trouver des parallélismes permettant d'en définir la dénotation. Il a été noté par Landman (1989) à la suite de Link (1983) que les caractéristiques des groupes diffèrent de celles des individus dont ils sont composés. Ces deux auteurs proposent l'idée selon laquelle, malgré le fait qu'un groupe soit composé d'unités atomiques, il constitue dans la langue une unité qui ne peut pas être décomposée. Cette hypothèse s'appuie sur des exemples tels que (31a) et (31b) qui illustrent le contraste en anglais entre le nom propre the Beatles qui est un nom de groupe et la coordination des quatre membres du groupe. Les exemples en (31) sont inspirés d'un exemple similaire fourni par Landman : dans la présente version, ce sont uniquement les noms propres qui ont été modifiés.

a. The Beatles were an English rock-band.

Anglais

'Les Beatles était un groupe de rock.'

b. $\quad *$ John, Paul, George and Ringo were an English rock-band.

Lit. 'John, Paul, George et Ringo étaient un groupe de rock.'

(adapté de Landman 1989)

Un SN qui désigne un groupe peut être sujet du prédicat 'être un groupe de rock', mais la coordination des noms des membres eux-mêmes ne peut pas l'être. Les définis pluriels ont une dénotation de somme, et non de groupe : eux non plus ne peuvent être sujets de ce type de prédicat, comme l'illustrent les exemples en (32).
a. \#Les gars étaient un groupe de rock.
b. \#The boys were a rock-band.

Anglais

Dans les exemples suivants, un SN contenant bann et un SN coordonné sont combinés avec le prédicat enn group sega 'un groupe de séga'. Les SN avec bann et les SN coordonnés ne sont pas compatibles avec ce type de prédicat.

a.

$\begin{array}{cccc}\text { *Bann garson enn group sega. } & \\ \text { BANN garçon un groupe séga }\end{array}$

Lit. 'Les garçons sont un groupe de séga.'

$\begin{array}{lllllll}\text { b. } & \text { *Sam, } & \text { Anil } & \text { ek } & \text { Raj } & \text { enn } & \text { group sega. } \\ & \text { S. } & \text { A. } & \text { et } & \text { R. } & \text { un } & \text { groupe séga }\end{array}$

Lit. 'Sam, Anil et Raj sont un groupe de séga.'

Ces résultats constituent la preuve que $b_{a n n}{ }^{\mathrm{PL}}+\mathrm{Nom}$ n'a pas la même dénotation qu'un $\mathrm{SN}$ dénotant un groupe. Les exemples en (33) s'opposent à ceux en (34) qui comprennent un SN ayant group comme tête et un nom propre de groupe (fictif). 


$$
\begin{aligned}
& \text { a. Sa group la enn group sega. } \\
& \text { DEM groupe DET un groupe séga }
\end{aligned}
$$

'Ce groupe est un groupe de séga.'

$$
\begin{array}{lll}
\text { b. Zanbalak } & \text { enn } & \text { group sega. } \\
\text { Z. } & \text { un } & \text { groupe séga }
\end{array}
$$

'Zanbalak est un groupe de séga.'

La problématique centrale dans cette section est la disponibilité de l'interprétation distributive pour les SN avec bann.

Il a été mentionné plus haut que les prédicats référant à des professions sélectionnent des SN à référence individuelle. Un de ces prédicats en CM est travay solda 'travailler comme soldat'. Afin de vérifier la disponibilité de la lecture distributive, je compare $b_{a n n}{ }^{\mathrm{PL}}+\mathrm{Nom}$ à un $\mathrm{SN}$ coordonné et à un $\mathrm{SN}$ dont la tête est group 'groupe'.

$$
\begin{array}{llll}
\text { Dan } & \text { sa } & \text { vilaz la, } \\
\text { dans dem village } & \text { det }
\end{array}
$$

\begin{tabular}{|c|c|c|c|c|}
\hline ?enn & group & garson & ti & travay \\
\hline
\end{tabular}

'Dans ce village,

$\begin{array}{lllll}\text { a. bann } & \text { garson ti } & \text { travay } & \text { solda. } \\ \text { BANN } & \text { garçon } & \text { PAST } & \text { travailler } & \text { solda }\end{array}$

'les garçons travaillaient comme soldats.'

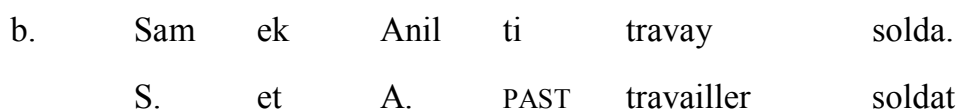

'Sam et Anil travaillaient comme soldats.'

'un groupe de garçons travaillait comme soldats.'

Le SN contenant bann et le SN coordonné sont tous deux compatibles avec le prédicat qui induit une lecture distributive. Tel n'est pas le cas pour le SN dont la tête est group. Le point d'interrogation indique une anomalie sémantique. Le contraste entre les nominaux de type 'groupe' et le SN avec bann indique que les SN avec bann ont une dénotation différente des groupes, et qu'ils dénotent peut-être des pluralités.

\section{$4 \quad$ Résumé}

Dans le cadre de cet article, j'ai formulé un certain nombre d'hypothèses. Les données suggèrent qu'il existe trois catégories syntaxiques bann : un nom lexical, un nom de quantité et un morphème de pluriel. Bann peut soit occuper la tête d'une projection nominale ou participer d'une expression de quantité dont je postule qu'il occupe la projection de quantité. Bann ${ }^{\mathrm{NQ}}$ (ou nom de quantité) et bann ${ }^{\mathrm{PL}}$ (pluralité) sont 
restreints au domaine nominal et opèrent une division du référent. Bann ${ }^{\mathrm{PL}}$ est la tête d'une projection fonctionnelle de quantité QtP : selon moi, il n'y a pas lieu de postuler une projection NumP en CM. J'ai aussi démontré que les SN contenant bann sont ouverts à la fois aux interprétations distributives et collectives.

\section{Références bibliographiques}

Abney, S. (1987). The English noun phrase in its sentential aspect. Thèse de doctorat. Cambridge, Mass. : MIT

Arrivé, M., Gadet, F. \& Galmiche M. (1986). La grammaire d'aujourd'hui. Paris : Flammarion.

Baker, P. (1972). Kreol, a description of Mauritian Creole. Londres: Hurst.

Baker, P. (2003). Réanalyse et grammaticalisation dans l'évolution du créole mauricien. In Kriegel, S. (éd.), Grammaticalisation et réanalyse : approches de la variation créole et française, Paris : CNRS, 111-141.

Bollée, A. (1977). Le créole français des Seychelles : esquisse d'une grammaire, textes, vocabulaire. Tübingen : Niemeyer.

Borer, H. (2005). In name only. Oxford: Oxford University Press.

Chaudenson, R. (2003). La créolisation : théorie, applications, implications. Paris : L’Harmattan.

Corbett, G. (2000). Number. Cambridge: Cambridge University Press.

Corne, C. (1970). Essai de grammaire du créole mauricien. Auckland : Linguistics Society of New Zealand, «Te Reo monographs».

Doetjes, J. (1997). Quantifiers and selection. La Haye : HIL.

Kwon, S.-N. \& Zribi-Hertz A. (2004). Number from a syntactic perspective : why plural marking looks 'truer' in French than in Korean. In Bonami O. \& Cabredo-Hofherr P. (éds.), Empirical issues in formal syntax and semantics 5 : Selected papers from CSSP 2003, http://www.cssp.cnrs.fr, 133-158.

Landman, F. (1989) Groups I. Linguistics and Philosophy, 12, 559-605.

Link, G. (1983) [2002] The Logical Analysis of Plurals and Mass Terms: A Lattice-theoretical Approach. In Portner P. \& Partee B. (éds.), Formal semantics: the essential readings. Oxford: Blackwell, 127-146.

Link, G. (1984)

Staudacher-Valliamée, G. (2004). Grammaire du créole réunionnais. Paris : Sedes/BUF.

Zribi-Hertz, A. \& Glaude, H. (2011). Bare NPs and deficient DPs in Haitian and French : from morphosyntax to referent construal. In Baptista M. \& Guéron J. (éds.), Noun phrases in creole languages: a multi-faceted approach, Amsterdam: Benjamins, 265-298.

$\begin{array}{llll}{ }^{1} \text { Abbrévisations utilisées : } & & \\ \text { ACC } & \text { accompli } & \text { IRR } & \text { irrealis } \\ \text { ANT } & \text { Antilles } & \text { PASS } & \text { passé } \\ \text { DEF } & \text { défini } & \text { PROG } & \text { progressif } \\ \text { FUT } & \text { futur } & \text { REU } & \text { Réunion } \\ \text { HAI } & \text { Haïti } & \text { SEY } & \text { Seychelles }\end{array}$

